\title{
Final Gleason Score Prediction Using Discriminant Analysis and Support Vector Machine Based on Preoperative Multiparametric MR Imaging of Prostate Cancer at 3T
}

\author{
Fusun Citak-Er, ${ }^{1}$ Metin Vural, ${ }^{2}$ Omer Acar, ${ }^{3}$ Tarik Esen, ${ }^{3,4}$ \\ Aslihan Onay, ${ }^{2}$ and Esin Ozturk-Isik ${ }^{5}$ \\ ${ }^{1}$ Department of Genetics and Bioengineering, Yeditepe University, Inönü Mah., Kayışdağı Cad, 26 Ağustos Yerleşimi, \\ Ataşehir, 34755 Istanbul, Turkey \\ ${ }^{2}$ Department of Radiology, VKF American Hospital, 34365 Istanbul, Turkey \\ ${ }^{3}$ Department of Urology, VKF American Hospital, 34365 Istanbul, Turkey \\ ${ }^{4}$ School of Medicine, Koç University, 34450 Istanbul, Turkey \\ ${ }^{5}$ Biomedical Engineering Institute, Boğaziçi University, Rasathane Cad, Kandilli Campus, Kandilli Mah., 34684 Istanbul, Turkey
}

Correspondence should be addressed to Fusun Citak-Er; fusun.er@gmail.com

Received 5 June 2014; Revised 10 September 2014; Accepted 12 September 2014; Published 2 December 2014

Academic Editor: Barıs Turkbey

Copyright (C) 2014 Fusun Citak-Er et al. This is an open access article distributed under the Creative Commons Attribution License, which permits unrestricted use, distribution, and reproduction in any medium, provided the original work is properly cited.

Objective. This study aimed at evaluating linear discriminant analysis (LDA) and support vector machine (SVM) classifiers for estimating final Gleason score preoperatively using multiparametric magnetic resonance imaging (mp-MRI) and clinical parameters. Materials and Methods. Thirty-three patients who underwent mp-MRI on a 3T clinical MR scanner and radical prostatectomy were enrolled in this study. The input features for classifiers were age, the presence of a palpable prostate abnormality, prostate specific antigen (PSA) level, index lesion size, and Likert scales of T2 weighted MRI (T2w-MRI), diffusion weighted MRI (DW-MRI), and dynamic contrast enhanced MRI (DCE-MRI) estimated by an experienced radiologist. SVM based recursive feature elimination (SVM-RFE) was used for eliminating features. Principal component analysis (PCA) was applied for data uncorrelation. Results. Using a standard PCA before final Gleason score classification resulted in mean sensitivities of $51.19 \%$ and $64.37 \%$ and mean specificities of $72.71 \%$ and $39.90 \%$ for LDA and SVM, respectively. Using a Gaussian kernel PCA resulted in mean sensitivities of $86.51 \%$ and $87.88 \%$ and mean specificities of $63.99 \%$ and $56.83 \%$ for LDA and SVM, respectively. Conclusion. SVM classifier resulted in a slightly higher sensitivity but a lower specificity than LDA method for final Gleason score prediction for prostate cancer for this limited patient population.

\section{Introduction}

Prostate cancer mortality rate has shown an increasing trend in adults over 40 years of age between 1950 and 2008 over the world according to the World Health Organization mortality database [1]. Prostate cancer screening is traditionally based on digital rectal exam (DRE) and prostate specific antigen (PSA) level measurement [2]. Patients with an elevated PSA level or with abnormal findings at DRE are candidates for further transrectal ultrasonography guided prostate biopsy (TRUS-Bx) [3]. However, TRUS-Bx of patients with raised
PSA level has a low sensitivity and a high false negative rate (15-30\%) due to the limitations of this test [4] and can lead to overdiagnosis and overtreatment of prostate cancers [2].

Multiparametric MRI has recently gained popularity as a tool for prostate cancer diagnosis and staging [5]. Multiparametric prostate MRI examination consists of anatomical and functional MR techniques, such as T1w-MRI, T2w-MRI, DW-MRI, and DCE-MRI, and MR spectroscopic imaging (MRSI). T1w-MRI can be used to detect intraprostatic bleeding, which is a common finding after previous biopsies [2]. $\mathrm{T} 2 \mathrm{w}$-MRI is optimal for depicting the zonal anatomy of 
the prostate. However, T2w-MRI has significant limitations for depicting cancer in transition zone, because benign prostatic hyperplasia nodules also can have low signal intensity on T2w-MR images depending on the size of stromal component. In addition, many benign conditions such as inflammation, biopsy related hemorrhage, post-radiation therapy fibrosis, and changes after hormone deprivation therapy may be seen as hypointense on T2w-MR images in the peripheral zone. DW-MRI gives information about cellular density through estimating the diffusivity of water molecules, and a decreased free diffusivity of water is seen in prostate carcinomas due to their dense cellularity [2]. Such restriction can be quantitatively evaluated by apparent diffusion coefficient (ADC) maps. In addition, the ADC values have negative correlation with the Gleason score of prostate carcinoma [6].

DCE-MRI is a noninvasive technique that collects information on the tumor angiogenesis. Prostate cancers typically show a steeper wash-in slope, higher peak enhancement, and rapid wash-out compared to healthy prostate tissue [2]. One of the major limitations of DCE-MRI is its nonspecificity. Similarly, prostatitis in the peripheral zone and BPH nodules in the central gland can show earlier and more pronounced enhancement than healthy prostate tissue [7].

Several studies have shown the diagnostic power of multiparametric MRI for prostate cancer. DCE-MRI combined with MRSI was shown to have $93.7 \%$ sensitivity and $90.7 \%$ specificity for detecting tumor foci in 150 prostate cancer patients [8]. Other studies have shown that combined MRSI and DW-MRI improved prostate cancer detection $[9,10]$. Roy et al. reported a specificity of $84.3 \%$ for prostate cancer detection before needle biopsy based on DW-MRI, T2wMRI, and DCE-MRI [11].

Gleason system is commonly used for grading prostate cancer [12]. The tissue obtained either by a biopsy or after a radical prostatectomy is graded from one to five, where a higher grade indicates more aggressiveness. The Gleason score is then calculated as the sum of the most and the second most predominant Gleason grades within the tissue section, and it ranges from two to ten [13]. Predicting the final Gleason score based on preoperative multiparametric MRI through a computer-aided diagnosis (CADx) system has been an ongoing interest. Puech et al. designed a CADx system that successfully determined five-level malignancy suspicion score based on wash-in and wash-out slope values of prostate cancer foci [14]. Sung et al. showed that a CADx system based on nonlinear support vector machine (SVM) outperformed the diagnostic ability of single DCE-MRI parameters and T2w-MRI by a $89 \%$ sensitivity and a $89 \%$ specificity in the peripheral zone (PZ) [15]. Support vector machine was also used to classify magnetic resonance spectra of prostate in order to assist prostate cancer localization [16]. Poulakis et al. combined PSA, biopsy Gleason score, and magnetic resonance imaging findings for prostate cancer staging using an artificial neural network [17]. Linear discriminant analysis was applied to analyze the power of the 10th percentile and average apparent diffusion coefficient (ADC) values, T2wMRI signal intensity histogram skewness, and Tofts $K^{\text {trans }}$ for the differentiation of prostate cancer from normal tissue
[18]. Niaf et al. compared nonlinear SVM, linear discriminant analysis, k-nearest neighbors, and naïve Bayes classifiers to determine an optimal CADx scheme to differentiate malignant tissue from suspicious or nonmalignant tissue at the peripheral zone [19].

In this study, a computer-aided diagnosis system that combined clinical and multiparametric MR findings was developed to predict preoperatively the final Gleason score of prostate cancers. Although various machine learning algorithms have been tested for the prediction and classification of prostate cancer and they mainly differed in the selection of the predictive parameters, according to our knowledge, the 5-point Likert scales of prostate MR images have not been previously evaluated. The main aim was to build a CADx model based on the 5-point Likert scale for multiparametric MRI data classification of prostate cancer in this study. Linear discriminant analysis and support vector machine classifiers were compared for their classification performances after a standard or a Gaussian kernel principal component analysis. Additionally, this work evaluated the contributions of the predictive parameters on prostate cancer malignancy detection by employing an SVM based recursive feature elimination and utilized the kernel trick to enhance the performance of classifiers.

\section{Materials and Methods}

2.1. Subjects. Thirty-three prostate cancer patients (mean ( \pm std.) age $=61.5 \pm 5.9$, range $=46-71$ ), who subsequently underwent radical prostatectomy, were included in this study. The institutional research committee approved this retrospective study. Routine clinical examination included digital rectal examination to detect the presence of a palpable prostate abnormality and serum PSA level measurement. Index lesion size was measured based on mp-MRI, and Gleason scores were determined based on the pathologic analysis of radical prostatectomy specimen.

\subsection{MRI Data Acquisition and Feature Extraction. All} patients were scanned on a 3T clinical MRI scanner (Magnetom Skyra, Siemens AG, Erlangen, Germany), using sixteenchannel phased array body coil. Before MR imaging, all patients were injected intramuscularly $20 \mathrm{mg}$ of butylscopolamine (Buscopan; Boehringer, Germany) to suppress bowel peristalsis. Multiparametric MR imaging protocol included 2D T2w-MRI, DW-MRI, and DCE-MRI. T2w-MRI was obtained in three orthogonal planes using T2w turbo spin echo (TSE) sequence. DW-MRI was obtained in axial orientation using a spin echo-echo planar imaging (SE-EPI) sequence with six $b$ values $(0,50,250,500,800$, and $1000 \mathrm{~s} / \mathrm{mm}^{2}$ ) and computed DW MR images were generated for $b$-values of 1200 and $1500 \mathrm{~s} / \mathrm{mm}^{2}$. Restriction of diffusion was quantified by the apparent diffusion coefficient (ADC) value. DCE-MRI was obtained using a fast 3D T1-weighted (T1-VIBE) gradient echo sequence in axial orientation. Each volumetric acquisition of the DCE sequence had an acquisition time of 7 seconds. A total of 35 contrast-enhanced 
acquisitions were performed. Perfusion curves were generated by using the image processing tools of an MRI CAD system (Dynacad; Invivo, Birmingham, MI). All MR images were interpreted by an experienced radiologist (14 years of experience in abdominal MRI and 4 years in prostate mpMRI), who was informed about the clinical findings of the patients. Low signal on T2w-MRI, low apparent diffusion coefficient $(\mathrm{ADC})$ values $(<1000)$, high signal intensity on high $b$ value imaging $(\geq 800)$, and types $2-3$ enhancement curve after contrast administration were interpreted as the main radiological criteria for an underlying prostate malignancy. ADC images were generated on the MRI scanner console. A Likert score was designated for T2w-MRI, DWMRI, and DCE-MRI within each region of interest according to a 5-point scale (i.e., the presence of clinically significant cancer is as follows: 1 = "extremely unlikely", 2 = "unlikely", 3 = "equivocal", 4 = "likely", and 5 = "extremely likely") based on ESUR guidelines [20].

The age of the patient, the presence or absence of a palpable prostate abnormality from digital rectal examination findings, PSA level, index lesion size based on mp-MRI, and Likert scales of T2w-MRI, DW-MRI, and DCE-MRI were used as the predictors. In this study, rather than all tumor foci, the index lesions have been evaluated. The index lesion was considered to be the largest lesion with a high Gleason score. In our study dataset, largest lesions had higher Gleason scores than other tumor foci. Gleason scores $3+3(n=3)$ and $3+4$ $(n=17)$ were defined as low-grade $(L)$ [21], whereas Gleason scores $4+3(n=7), 4+4(n=2), 4+5(n=2)$, and $5+4(n=2)$ were defined as high-grade $(H)$ in this small patient population. The age of the patients was mapped into binary values, where patients who were older than 65 [22] were considered as old (1) and otherwise as young (0).

A computer-aided diagnosis (CADx) system was designed for interpreting multiparametric prostate cancer MRI data. Feature elimination using support vector machine based recursive feature elimination, data uncorrelation using principal component analysis (PCA) with or without a Gaussian kernel, classification, and evaluation were the major stages of this CADx. The performance of two classification algorithms, which were the linear discriminant analysis and linear SVM, was assessed for predicting the final Gleason score of radical prostatectomy specimen based on the chosen seven clinical and radiological predictors.

2.3. Feature Elimination. Support vector machine based recursive feature elimination [23] was used to eliminate the least important feature before subsequent analytical operations for assessing the contribution of the features. As a first step, a support vector machine was trained using the dataset defined as

$$
S=\left\{\left(x_{i}, y_{i}\right)\right\}_{i=1, n}, \quad x_{i} \in R^{f}, y_{i} \in\{-1,+1\},
$$

where $f$ is the number of features, $n$ is the number of samples, $x$ are the feature vectors of size $f$, and $y$ are the outputs of the corresponding feature vector $x$. The possible values for $y$ were $(-1)$ and $(+1)$, which indicated the low and high Gleason score groups, respectively.
As a second step of the feature elimination, the Lagrange multipliers are used to calculate the scores of the features individually. The score of a feature, $j$, is the square of the sum of the product of $\alpha, y$ and the value of that feature for all support vectors, formulated as

$$
r_{j}=\left(\sum_{i=1}^{k} \alpha_{i} y_{i} x_{i, j}\right)^{2},
$$

where $\alpha_{i}, y_{i}$, and $x_{i, j}$ are the Lagrange multiplier, the output, and the value of the $j$ th feature of the $k$ number of support vectors, respectively. Finally, the feature having the minimum score can be eliminated. In this study, the least important feature was eliminated at each step.

2.4. Data Uncorrelation. After feature elimination, standard and kernel principal component analysis (kernel PCA) was used to uncorrelate the data [24]. Standard principal component analysis aims to find a new coordinate system, which is composed of a set of orthogonal vectors, called the "principal components" (PC). Kernel PCA is the nonlinear form of PCA that nonlinearly maps the dataset to a higher dimensional feature space via kernel trick, which uses kernel matrix instead of the covariance matrix unlike standard PCA [25]. Since standard PCA produces linear feature space, it is not suitable for complex data distributions. Kernel PCA outperforms standard PCA in most cases [26].

In this study, Gaussian kernel PCA was implemented in MATLAB using kernel PCA and pre-image reconstruction software [27]. The data was placed in an $n \times f$ data matrix, where the rows represented $n$ number of samples and columns were $f$ number of features. Instead of calculating the covariance matrix, the data was transformed into an $n \times$ $n$ dimensional high feature space with the Gaussian kernel function as

$$
K(x)=\exp \left(\frac{-\|x\|^{2}}{2 \sigma^{2}}\right),
$$

where $x$ is the dataset and $\sigma$ is a performance regularization constant of the kernel. Then, standard PCA was implicitly applied to the kernel matrix. Therefore, the original dataset was projected into the coordinate system of the eigenvectors, which is a nonlinear representation of the original dataset. The scree graph [24] was used to determine the cut-off point for the number of principal components to represent the data.

\subsection{Classification Based on Discriminant Analysis. Linear} discriminant analysis searches for a vector, $w^{T}$, called a discriminant transformation function that results in the minimum intraclass and the maximum interclass distances when a data is projected onto it [28]. Given a dataset $X$ as

$$
\begin{aligned}
X=\left\{\left(x_{i}, y_{i}\right)\right\}_{i=1, n}, & x_{i} \in R^{f}, y_{i} \in\{0,+1\}, \\
y_{i} & =v \text { then } y_{i} \in C_{v},
\end{aligned}
$$

a projection $z$ of $x$ onto the vector $w$ can be defined as

$$
z=w^{T} x
$$




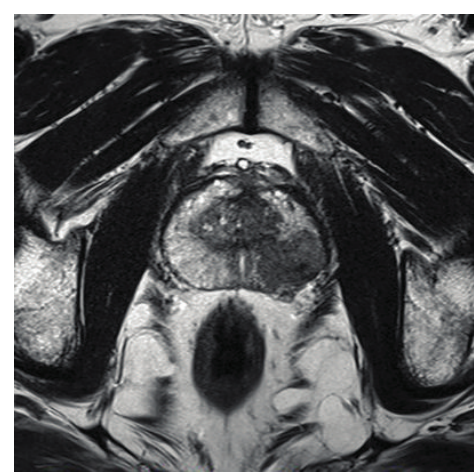

(a)

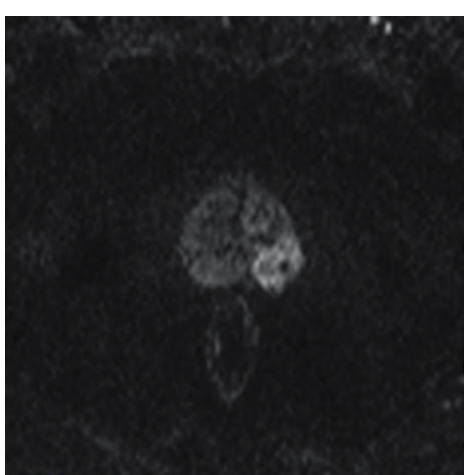

(b)

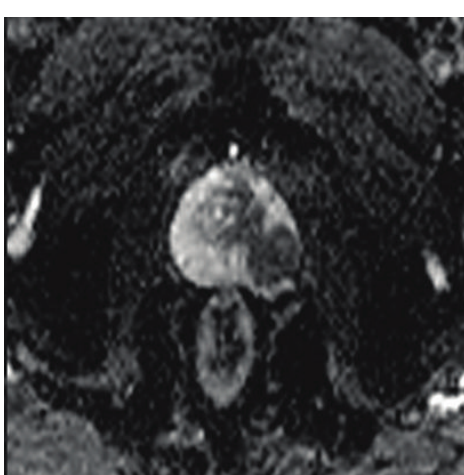

(c) (c)

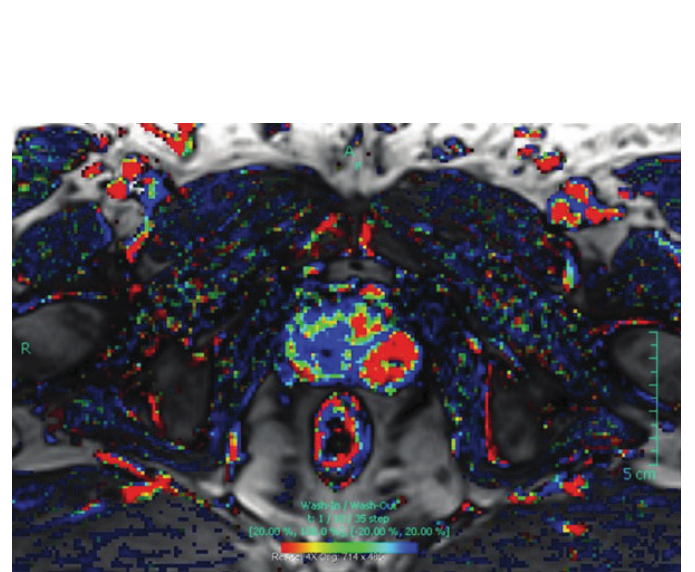

(d)

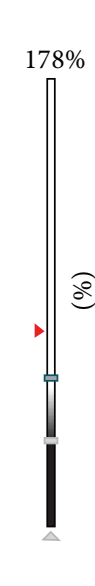

$$
\begin{array}{r}
500 \\
400 \\
300 \\
200 \\
100 \\
-100 \\
0
\end{array}
$$

Wash-in/wash-out

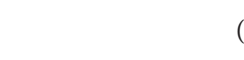


TABLE 1: The number of subjects for the two binary features (age and DRE), and the mean ( \pm std.) values for the other five features grouped by low and high Gleason score patients.

\begin{tabular}{|c|c|c|c|c|}
\hline \multirow{3}{*}{ Age } & \multicolumn{2}{|c|}{ Low-grade $(n=20)$} & \multicolumn{2}{|c|}{ High-grade $(n=13)$} \\
\hline & $(<65)$ & 16 & $(<65)$ & 7 \\
\hline & $(\geq 65)$ & 4 & $(\geq 65)$ & 6 \\
\hline \multirow{2}{*}{ DRE } & No & 17 & No & 8 \\
\hline & Yes & 3 & Yes & 5 \\
\hline PSA & \multicolumn{2}{|c|}{$8.42 \pm 12.92$} & \multicolumn{2}{|c|}{$9.91 \pm 9.35$} \\
\hline Index lesion size & \multicolumn{2}{|c|}{$1.64 \pm 1.78$} & \multicolumn{2}{|c|}{$2.23 \pm 1.64$} \\
\hline Likert scale of T2w-MRI & \multicolumn{2}{|c|}{$3.90 \pm 0.72$} & \multicolumn{2}{|c|}{$4.00 \pm 0.71$} \\
\hline Likert scale of DW-MRI & \multicolumn{2}{|c|}{$4.55 \pm 0.60$} & \multicolumn{2}{|c|}{$4.69 \pm 0.48$} \\
\hline Likert scale of DCE-MRI & \multicolumn{2}{|c|}{$3.55 \pm 1.47$} & \multicolumn{2}{|c|}{$4.62 \pm 0.51$} \\
\hline
\end{tabular}

TABLE 2: The scores calculated by SVM-RFE in three iterations.

\begin{tabular}{lccc}
\hline & 1st iteration & 2nd iteration & 3rd iteration \\
\hline Age & 32.94 & $\mathbf{3 5 . 2}$ & - \\
DRE & $\mathbf{2 1 . 6 5}$ & - & $\mathbf{1}$ \\
PSA & 18451.23 & 18807.95 & $\mathbf{1 8 9 9 2 . 8 6}$ \\
The size of lesion & 881.93 & 900.45 & $\mathbf{9 3 0 . 0 7}$ \\
T2W-MRI Likert scale & 2899.26 & 3029.82 & 3179.27 \\
DW-MRI Likert scale & 4055.23 & 4232.81 & 4423.32 \\
DCE-MRI Likert scale & 3857.99 & 4027.87 & 4242.97 \\
\hline
\end{tabular}

${ }^{*}$ The eliminated features are marked as bold, which are then not included in the subsequent iterations.

Support vector machine based feature elimination was repeated three times, and the feature having the least score was eliminated from the analysis at each iteration. Table 2 shows the scores of features at each iteration, where the features having the least score within each iteration are marked as bold. Digital rectal exam findings, age, and the lesion size were eliminated from the classification after three iterations.

Then, a standard or a Gaussian kernel PCA was applied to uncorrelate the data after each iteration of the SVM-RFE. The sigma $(\sigma)$ parameter of Gaussian kernel and the size of the remaining dimension were considered separately at each iteration. Figure 2 shows the results of data uncorrelation via Gaussian kernel PCA after each iteration of SVM-RFE. The scree plots of the eigenvalues versus the principal components with the corresponding cumulative percent variances and the first three principal components of the uncorrelated data are shown. The principal component located at the elbow of the scree plot was selected as the cut-off point for the total number of principal components. A cut-off point that keeps $80 \%$ to $90 \%$ of the data variance was selected [30].

Table 3 shows the averages and confidence intervals of thirty iterations of the accuracies, sensitivities, and specificities for the four combinations of Gaussian kernel PCA and standard PCA with LDA classifier and SVM classifier. All accuracy and sensitivity values were statistically significantly different between the Gaussian kernel PCA and standard PCA based on a Mann-Whitney rank sum test $(\alpha<0.002)$. The specificities of LDA methods were not statistically significantly different when applied after two kinds of PCA methods. The performance measurements of LDA and SVM methods were statistically significantly different when standard PCA and SVM-RFE were applied $(\alpha<0.002)$. Gaussian kernel PCA significantly outperformed standard PCA in all cases. Feature elimination with SVMRFE increased the classification performance of SVM in terms of accuracy and sensitivity.

\section{Discussion}

Multiparametric MRI has been reported to have diagnostic value for prostate cancer. The value of ADC has been found to be strongly correlated with the aggressiveness of prostate cancer $[6,31,32]$ and the tumor growth rate [33]. It was reported that the $\mathrm{T} 2{ }^{*}$ values of the cancerous prostatic regions were significantly lower than those of the benign prostatic regions [34]. It was proposed that quantitative diffusion tensor imaging (DTI) analysis can be used to discriminate prostate cancer from normal tissue [35]. Transition zone entropy of T2 and T1 weighted images obtained by application of spatial filters was proposed as a new promising diagnostic feature for the development of mp-MRI based CADx systems [36]. Vos et al. concluded that the combinational use of DW-MRI and T2w-MRI is sufficient for the assessment of prostate cancer aggressiveness in the peripheral zone, while DCE-MRI and MRSI should be additionally considered for discrimination of indolent and high-grade prostate cancers [37]. 

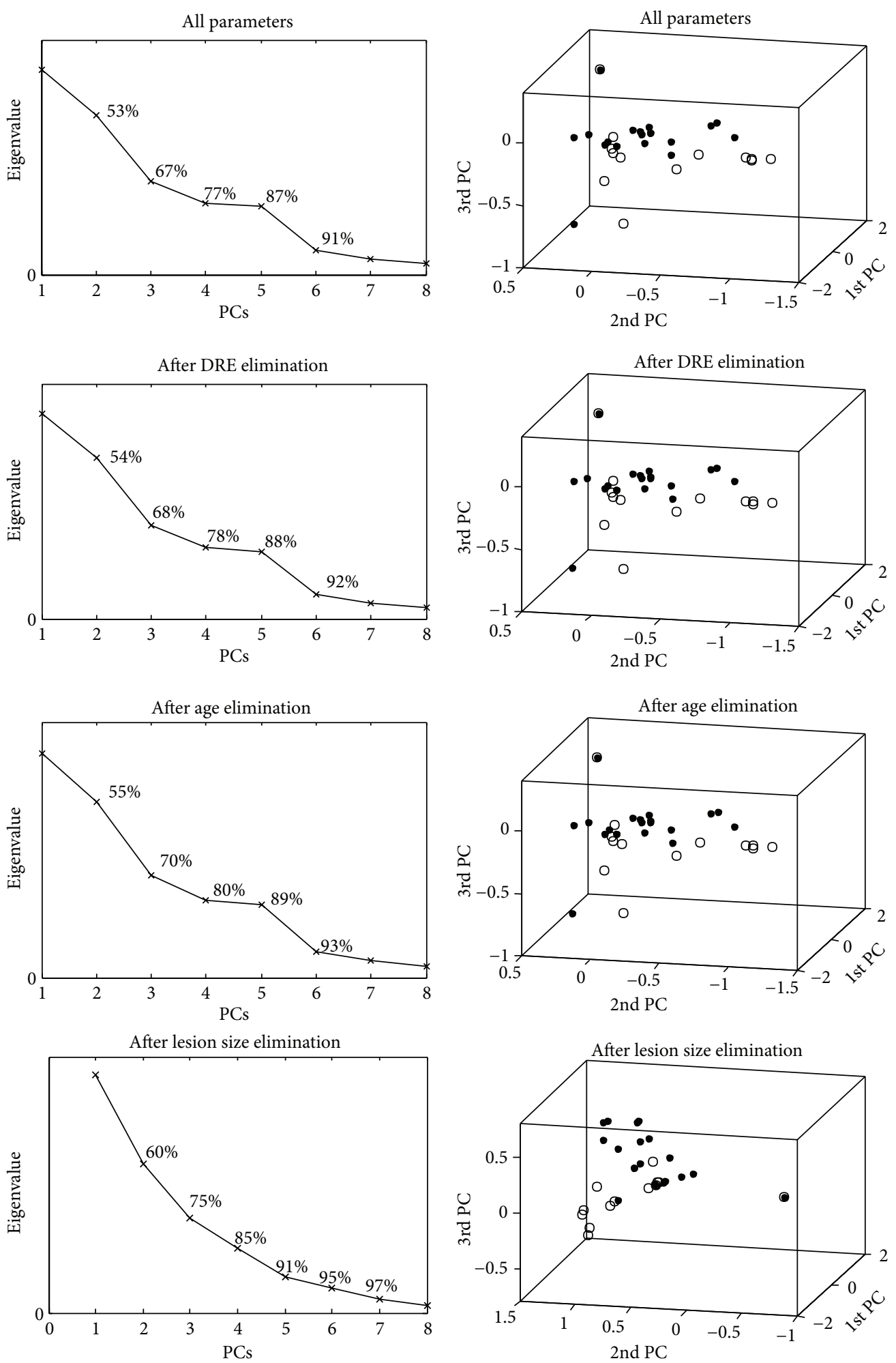

(a)

(b)

FIGURE 2: The results of data uncorrelation via Gaussian kernel PCA after each iteration of SVM-RFE. The scree plots of the eigenvalues versus principal components (PCs) (a) and the distribution of the first three principal components at each data point (b) are given. The high-grade data are labeled as circles, while the low-grade data are labeled as dots. 
TABLE 3: The averages and confidence intervals of accuracy, sensitivity, and specificity values for the combinations of two kinds of classifiers and two kinds of PCA averaged after thirty iterations.

\begin{tabular}{|c|c|c|c|c|c|c|c|c|}
\hline & & & Accuracy [CI] (\%) & & Sensitivity [CI] (\%) & & Specificity $[\mathrm{CI}](\%)$ & \\
\hline \multirow{4}{*}{ All parameters } & \multirow{2}{*}{ SVM } & Kernel PCA & 76.83 [75.77-77.90] & \multirow{2}{*}{$*$} & $83.38[81.77-85.00]$ & \multirow[b]{2}{*}{$*$} & $68.36[65.88-70.84]$ & \multirow{4}{*}{$*$} \\
\hline & & Standard PCA & $52.03[50.52-53.53]$ & & $58.16[55.94-60.38]$ & & $47.87[43.93-51.8]$ & \\
\hline & \multirow{2}{*}{ LDA } & Kernel-PCA & $76.56[75.42-77.70]$ & \multirow{2}{*}{$*$} & 81.59 [79.87-83.31] & \multirow{2}{*}{$*$} & $70.09[67.66-72.52]$ & \\
\hline & & Standard PCA & 60.03 [58.88-61.17] & & $55.72[53.98-57.45]$ & & 67.55 [64.19-70.91] & \\
\hline \multirow{4}{*}{ DRE eliminated } & \multirow{2}{*}{ SVM } & Kernel PCA & $76.17[75.12-77.21]$ & \multirow{2}{*}{$*$} & $84.59[83.22-85.96]$ & \multirow{2}{*}{ * } & $66.07[63.67-68.46]$ & \multirow{4}{*}{ * } \\
\hline & & Standard PCA & $51.94[50.17-53.72]$ & & $58.24[55.92-60.55]$ & & $46.11[41.70-50.53]$ & \\
\hline & \multirow{2}{*}{ LDA } & Kernel PCA & $75.36[74.01-76.71]$ & \multirow{2}{*}{$*$} & $82.59[80.84-84.34]$ & \multirow{2}{*}{$*$} & $66.27[63.75-68.79]$ & \\
\hline & & Standard PCA & 60.11 [59.09-61.13] & & $55.28[53.61-56.94]$ & & $67.14[63.83-70.46]$ & \\
\hline \multirow{4}{*}{ Age eliminated } & \multirow{2}{*}{ SVM } & Kernel PCA & $75.97[75.07-76.88]$ & \multirow{2}{*}{$*$} & $85.12[83.73-86.51]$ & \multirow{2}{*}{$*$} & $64.15[61.57-66.72]$ & \multirow{4}{*}{$*$} \\
\hline & & Standard PCA & 56.03 [53.97-58.09] & & $67.85[65.64-70.07]$ & & $40.55[35.62-45.49]$ & \\
\hline & \multirow{2}{*}{ LDA } & Kernel PCA & 76.33 [75.10-77.56] & \multirow{2}{*}{$*$} & $83.95[82.10-85.80]$ & \multirow{2}{*}{$*$} & 66.48 [63.27-69.69] & \\
\hline & & Standard PCA & 57.50 [56.11-58.89] & & 51.81 [49.34-54.28] & & $65.01[62.26-67.77]$ & \\
\hline \multirow{4}{*}{ Index Lesion Size Eliminated } & \multirow{2}{*}{ SVM } & Kernel PCA & $75.31[74.19-76.42]$ & \multirow{2}{*}{$*$} & $87.88[86.31-89.45]$ & \multirow{2}{*}{ * } & $56.83[53.94-59.72]$ & \multirow{2}{*}{ * } \\
\hline & & Standard PCA & $53.64[52.52-54.76]$ & & $64.37[62.52-66.22]$ & & $39.90[36.66-43.15]$ & \\
\hline & \multirow{2}{*}{ LDA } & Kernel PCA & $76.75[75.64-77.86]$ & \multirow{2}{*}{ * } & $86.51[84.94-88.08]$ & \multirow[b]{2}{*}{ * } & $64.00[61.22-66.77]$ & \\
\hline & & Standard PCA & $59.69[58.36-61.03]$ & & $51.20[48.89-53.50]$ & & $72.71[69.60-75.81]$ & \\
\hline
\end{tabular}

${ }^{*}$ Statistically significantly different $(\alpha<0.05 / 24)$.

Many subsets of mp-MRI features have been proposed as the candidate predictors for a computer-aided prostate cancer diagnosis system in the literature. Grey-level histogram of T2w-MRI, ADC value, and semi-quantitative features extracted from DCE curves were used as the features of a prostate cancer CADx system, where sequential forward selection (SFS) feature selection algorithm was employed to determine the best combination of these features [38]. Several combinations of features extracted from DTI [39] and DCE-MRI [14, 39-41] were used for detection and grading of prostate cancer. A logistic regression model was fitted using ADC, elevated choline peaks in MR spectroscopy, increased perfusion, and malignant wash-out parameters for prostate cancer detection [42]. The ratios of sum of total choline, spermine, and creatine (CSC) to citrate (CSC/Cit) and total choline to citrate (tCho/Cit) were used as predictors of two different CADx models to assess the aggressiveness of prostate cancer, with resultant sensitivities of $87 \%$ and $81 \%$, respectively [43]. Support vector machines were used to build an image-based computer-aided diagnosis system, which creates a cancer probability map of prostate cancer based on DTI and DCE-MRI [39]. The combination of 10th percentile of ADC, average ADC, and T2-weighted skewness was used to build a CADx system for differentiating normal and tumor tissues in the prostate [18].

On the other hand, mp-MRI approach has some limitations. Some of these MRI modalities might not be available for each patient. Some solutions were proposed to estimate missing DTI and DCE parameters [44]. Also, T2w-MR imaging has been reported as being difficult to incorporate into a CAD system due to its sensitivity to patient motion [45]. To our knowledge, the 5-point Likert scales of prostate MR images have not been previously used as predictors of a CADx system for the pre-operative prediction of final Gleason score.
The Likert scale encompasses the mp-MRI information in a more standardized way, which might result in less variability between different sites. Furthermore, clinical findings were also incorporated in this study.

The main limitation of this study was the small patient data size. Although the study has been ongoing for the past two years, only thirty-three patients, who later underwent a radical prostatectomy, have been successfully recruited. The main reason for low specificity was the lower number of highgrade data $(n=13)$ than the low-grade $(n=20)$. As a result, even a small number of false positives resulted in a noticeable loss in specificity.

Additionally, a kernel PCA was employed to extract nonlinear relationship between predictors. The most suitable kernel was searched to improve the efficiency of PCA, and the best results were achieved when a Gaussian kernel was employed. For this small dataset, Gaussian kernel PCA worked noticeably better than the standard PCA. However, the computational complexity of kernel PCA is higher than the standard PCA. Kernel PCA processes an $n \times n$ kernel matrix, where $n$ is the size of the training vector, while standard PCA works on an $f \times f$ covariance matrix, where $f$ is the number of features of the training vector. Several enhancements of kernel PCA have been proposed in the literature to solve this issue, which will be considered in the future studies as the size of the data increases [26]. More suitable kernels for PCA should also be investigated in future studies.

Selecting the best subset of features in a large hypothesis space defined by all possible combinations of features is a well-known issue in machine learning. The main advantage of the feature elimination is to avoid overfitting, which means a perfect fit of the decision function for the training dataset, but a failed fit for the subsequent test datasets. 
In this study, SVM-RFE was used to eliminate redundant features for a better classification and to avoid overfitting. The classifiers often perform better after feature elimination [28]. However, there is a trade-off between feature elimination and classification. If the classifier is sufficiently strong, then feature elimination might not be necessary. In this study, mean sensitivities of $81.59 \%, 83.38 \%, 55.72 \%$, and $58.16 \%$ and mean specificities of $70.09 \%, 68.36 \%, 67.55 \%$, and $47.87 \%$ were attained for LDA with Gaussian kernel PCA, SVM with Gaussian kernel PCA, LDA with standard PCA, and SVM with standard PCA, respectively, when feature elimination was not performed. The mean sensitivities of the classifiers with Gaussian kernel PCA were statistically significantly higher while the mean specificities of them were slightly lower when all feature elimination iterations were applied. It was observed that the specificity of SVM was more affected from feature elimination than LDA; however, it was essential to apply feature elimination for a higher sensitivity and for assessing the contribution of each feature for classification.

\section{Conclusion}

The application of a Gaussian kernel PCA increased the performances of both classification models for an accurate prediction of final Gleason score based on clinical findings and preoperative multiparametric MR imaging for this limited patient population, while exploiting the complicated relationship between features. Linear discriminant analysis provided a slightly higher specificity than the SVM method, which can be related to the small intraclass distance. It was observed that mp-MRI features were more important than clinical features based on SVM-RFE scores, and applying feature elimination procedure increased the classification performances of the models. One novelty of this study is modeling a prediction system based on Likert scales of mpMRI rather than a set of mp-MRI features, which might be a more standardized way of assessing mp-MRI data. Future studies will investigate the role of Likert scales of preoperative MR imaging parameters in final Gleason score prediction in a larger patient population. The analytical results of a CADx system based on multiparametric MRI might help radiologists in clinical decision making.

\section{Conflict of Interests}

The authors declare that there is no conflict of interests regarding the publication of this paper.

\section{Acknowledgments}

This study was supported in part by TUBITAK Career Development Grant 112E036 and EU Marie Curie IRG Grant FP7-PEOPLE-RG-2009 256528.

\section{References}

[1] T. Matsuda and A. Matsuda, "Time trends in prostate cancer mortality between 1950 and 2008 in Japan, the USA and Europe based on the who mortality database," Japanese Journal of Clinical Oncology, vol. 41, no. 12, Article ID hyr177, p. 1389, 2011.

[2] T. Penzkofer and C. M. Tempany-Afdhal, "Prostate cancer detection and diagnosis: the role of MR and its comparison with other diagnostic modalities-a radiologist's perspective," NMR in Biomedicine, vol. 27, no. 1, pp. 3-15, 2014.

[3] M. N. Simmons, R. K. Berglund, and J. S. Jones, "A practical guide to prostate cancer diagnosis and management," Cleveland Clinic Journal of Medicine, vol. 78, no. 5, pp. 321-331, 2011.

[4] M. B. Garnick, "Prostate cancer: screening, diagnosis, and management," Annals of Internal Medicine, vol. 118, no. 10, pp. 804-818, 1993.

[5] B. Turkbey and P. L. Choyke, "Multiparametric MRI and prostate cancer diagnosis and risk stratification," Current Opinion in Urology, vol. 22, no. 4, pp. 310-315, 2012.

[6] H. Zhou, R. R. Hallac, Q. Yuan et al., "Correlating multiparametric MRI with Gleason score in human prostate," in International Society of Magnetic Resonance in Medicine Annual Conference, Milan, Itay, May 2014.

[7] S. Verma, B. Turkbey, N. Muradyan et al., "Overview of dynamic contrast-enhanced MRI in prostate cancer diagnosis and management," The American Journal of Roentgenology, vol. 198, no. 6, pp. 1277-1288, 2012.

[8] V. Panebianco, A. Sciarra, M. Ciccariello et al., "Role of magnetic resonance spectroscopic imaging ([(1)H]MRSI) and dynamic contrast-enhanced MRI (DCE-MRI) in identifying prostate cancer foci in patients with negative biopsy and high levels of prostate-specific antigen (PSA)," Radiologia Medica, vol. 115, no. 8, pp. 1314-1329, 2010.

[9] S. A. Reinsberg, G. S. Payne, S. F. Riches et al., "Combined use of diffusion-weighted MRI and $1 \mathrm{H}$ MR spectroscopy to increase accuracy in prostate cancer detection," The American Journal of Roentgenology, vol. 188, no. 1, pp. 91-98, 2007.

[10] S. F. Riches, G. S. Payne, V. A. Morgan et al., "MRI in the detection of prostate cancer: combined apparent diffusion coefficient, metabolite ratio, and vascular parameters," The American Journal of Roentgenology, vol. 193, no. 6, pp. 1583-1591, 2009.

[11] C. Roy, R. Pasquali, A. Matau, G. Bazille, and H. Lang, "The role of diffusion 3-Tesla MRI in detecting prostate cancer before needle biopsy: multiparametric study of 111 patients," Journal de Radiologie, vol. 91, no. 11, part 1, pp. 1121-1128, 2010.

[12] D. F. Gleason, "Histologic grading of prostate cancer: a perspective," Human Pathology, vol. 23, no. 3, pp. 273-279, 1992.

[13] R. Montironi, F. R. Path, and R. Mazzucchelli, "Gleason grading of prostate cancer. Contemporary approach., Pathologica., vol. 97, no. 4, p. 164, 2005.

[14] P. Puech, N. Betrouni, N. Makni, A. S. Dewalle, A. Villers, and L. Lemaitre, "Computer-assisted diagnosis of prostate cancer using DCE-MRI data: design, implementation and preliminary results," International Journal of Computer Assisted Radiology and Surgery, vol. 4, no. 1, pp. 1-10, 2009.

[15] Y. S. Sung, H.-J. Kwon, B.-W. Park et al., "Prostate cancer detection on dynamic contrast-enhanced MRI: computer-aided diagnosis versus single perfusion parameter maps," The American Journal of Roentgenology, vol. 197, no. 5, pp. 1122-1129, 2011.

[16] S. Parfait, P. M. Walker, G. Créhange, X. Tizon, and J. Mitéran, "Classification of prostate magnetic resonance spectra using Support Vector Machine," Biomedical Signal Processing and Control, vol. 7, no. 5, pp. 499-508, 2012. 
[17] V. Poulakis, U. Witzsch, R. de Vries et al., "Preoperative neural network using combined magnetic resonance imaging variables, prostate specific antigen and gleason score to predict prostate cancer stage," Journal of Urology, vol. 172, no. 4, pp. 1306-1310, 2004.

[18] Y. Peng, Y. Jiang, C. Yang et al., "Quantitative analysis of multiparametric prostate MR images: differentiation between prostate cancer and normal tissue and correlation with Gleason score: a computer-aided diagnosis development study," Radiology, vol. 267, no. 3, pp. 787-796, 2013.

[19] E. Niaf, O. Rouvière, F. Mège-Lechevallier, F. Bratan, and C. Lartizien, "Computer-aided diagnosis of prostate cancer in the peripheral zone using multiparametric MRI," Physics in Medicine and Biology, vol. 57, no. 12, pp. 3833-3851, 2012.

[20] J. O. Barentsz, J. Richenberg, R. Clements et al., "ESUR prostate MR guidelines 2012," European Radiology, vol. 22, no. 4, pp. 746757, 2012.

[21] K. D. Berg, M. A. Røder, K. Brasso, B. Vainer, and P. Iversen, "Primary Gleason pattern in biopsy Gleason score 7 is predictive of adverse histopathological features and biochemical failure following radical prostatectomy," Scandinavian Journal of Urology, vol. 48, no. 2, pp. 168-176, 2014.

[22] F. Abdollah, M. Sun, J. Schmitges et al., "Survival benefit of radical prostatectomy in patients with localized prostate cancer: estimations of the number needed to treat according to tumor and patient characteristics," The Journal of Urology, vol. 188, no. 1, pp. 73-83, 2012.

[23] I. Guyon, J. Weston, S. Barnhill, and V. Vapnik, "Gene selection for cancer classification using support vector machines," Machine Learning, vol. 46, no. 1-3, pp. 389-422, 2002.

[24] R. B. Cattell, "Citation classic-the Scree test for the number of factors," Current Contents/Social \& Behavioral Sciences, no. 5, p. $16,1983$.

[25] B. Schölkopf, A. J. Smola, and K. R. Müller, "Kernel principal component analysis," in Advances in Kernel Methods, pp. 327352, MIT Press, Cambridge, Mass, USA, 1999.

[26] T.-J. Chin and D. Suter, "Incremental kernel principal component analysis," IEEE Transactions on Image Processing, vol. 16, no. 6, pp. 1662-1674, 2007.

[27] Q. Wang, "Kernel principal component analysis and its applications in face recognition and active shape models," http://arxiv.org/abs/1207.3538.

[28] E. Alpaydın, Introduction to Machine Learning, MIT Press, Cambridge, Mass, USA, 2nd edition, 2004.

[29] C. Cortes and V. Vapnik, "Support-vector networks," Machine Learning, vol. 20, no. 3, pp. 273-297, 1995.

[30] I. T. Jolliffe, Principal component analysis, Springer, 2nd edition, 2002.

[31] R. Nagarajan, Z. Iqbal, B. Burns et al., "Maximum entropy reconstructed echo planar based spectroscopic imaging and diffusion weighted imaging in prostate cancer," in Proceedings of the International Society of Magnetic Resonance in Medicine Annual Conference, Milan, Italy, May 2014.

[32] M. C. Maas, A. J. Wright, K. M. Selnæs et al., "Prostate cancer localization with a multiparametric MR approach (PCaMAP): separating PCa from Benign tissue in a multi-center study," in International Society of Magnetic Resonance in Medicine Annual Conference, Milan, Italy, May 2014.

[33] V. Morgan, C. C. Parker, S. Giles, and N. de Souza, "Monitoring low-risk prostate cancer withdiffusion-weighted MRI: ADC and its relationship to growth rate," in Proceedings of the
International Society of Magnetic Resonance in Medicine Annual Conference, Milan, Italy, May 2014.

[34] C. Xiaoxi, L. Wu, Y. Dai, and J. Xu, "Feasibility and preliminary experience of quantitative $T 2^{*}$ mapping at $3.0 \mathrm{~T}$ for detection and assessment of aggressiveness of prostate cancer," in Proceedings of the International Society of Magnetic Resonance in Medicine Annual Conference, Milan, Italy, May 2014.

[35] A. A. Tonyushkin, S. S. Hedgire, P. F. Hahn et al., "Quantitative DTI tractography of prostate gland in prostate cancer patients," in Proceedings of the International Society of Magnetic Resonance in Medicine Annual Conference, Milan, Italy, May 2014.

[36] S. Punwani, S. Benigno, B. Ganeshan, A. Groves, and M. Emberton, "Textural entropy may help identify significant tumour within the prostatic transition zone on mp-MRI," in Proceedings of the Medicine Annual Conference, pp. 10-16, Milan, Italy, May 2014.

[37] E. K. Vos, T. Kobus, G. Litjens et al., "Multiparametric MR imaging for the assessment of prostate cancer aggressiveness at 3 Tesla," in Proceedings of the International Society of Magnetic Resonance in Medicine Annual Conference, pp. 10-16, Milan, Italy, 2014.

[38] C. Wang, S. Cheng, J. Hu et al., "Comparative study of four widely used classifiers for prostate cancer detection with multiparametric MRI," in International Society of Magnetic Resonance in Medicine Annual Conference, Milan, Italy, May 2014.

[39] M. Moradi, S. E. Salcudean, S. D. Chang et al., "Multiparametric MRI maps for detection and grading of dominant prostate tumors," Journal of Magnetic Resonance Imaging, vol. 35, no. 6, pp. 1403-1413, 2012.

[40] O. Starobinets, J. Simko, K. Kuchinsky et al., "Multiparametric MRI to differentiate high-risk from low-risk prostate cancer," in Proceedings of the International Society of Magnetic Resonance in Medicine Annual Conference, Milan, Italy, May 2014.

[41] P. C. Vos, T. Hambrock, C. A. Hulsbergen - Van De Kaa, J. J. Fütterer, J. O. Barentsz, and H. J. Huisman, "Computerized analysis of prostate lesions in the peripheral zone using dynamic contrast enhanced MRI," Medical Physics, vol. 35, no. 3, pp. 888899, 2008.

[42] A. D. Nicholson, V. A. Master, T. E. Powell et al., "Multiparametric prostate MRI-correlation of imaging findings with MRIguided biopsy results," in Proceedings of the International Society of Magnetic Resonance in Medicine Annual Conference, Milan, Italy, May 2014.

[43] G. J. Metzger, B. Fossen, P. J. Bolan et al., "Detection and grading of prostate cancer using model-based spectral fitting," in Proceedings of the International Society of Magnetic Resonance in Medicine Annual Conference, Milan, Italy, May 2014.

[44] H. A. Ashab, P. Kozlowski, R. Rohling et al., "Handling missing DCE data in prostate cancer detection using multiparametric MRI," in International Society of Magnetic Resonance in Medicine Annual Conference, Milan, Italy, May 2014.

[45] P. C. Vos, T. Hambrock, J. O. Barenstz, and H. J. Huisman, "Computer-assisted analysis of peripheral zone prostate lesions using T2-weighted and dynamic contrast enhanced T1weighted MRI," Physics in Medicine and Biology, vol. 55, no. 6, pp. 1719-1734, 2010. 


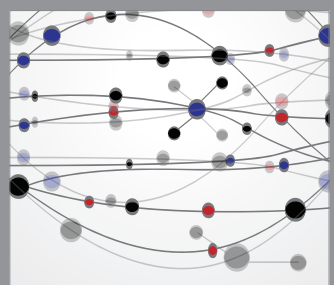

The Scientific World Journal
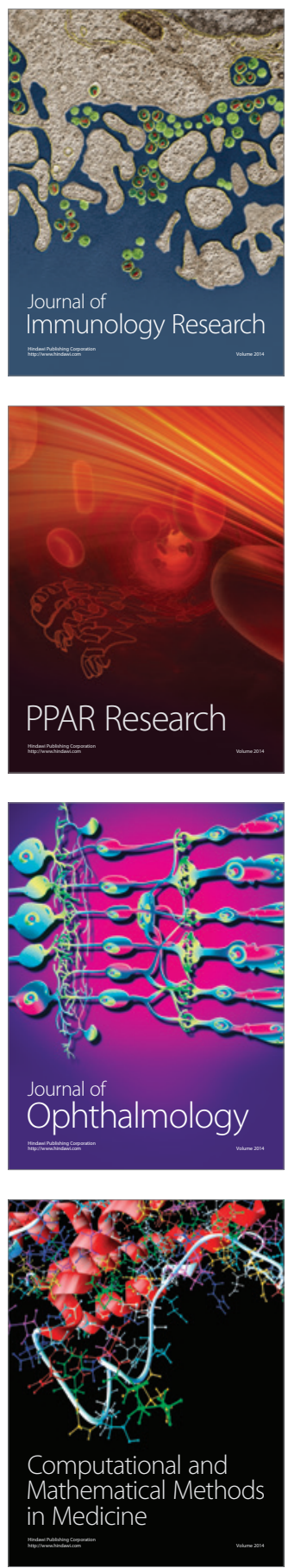

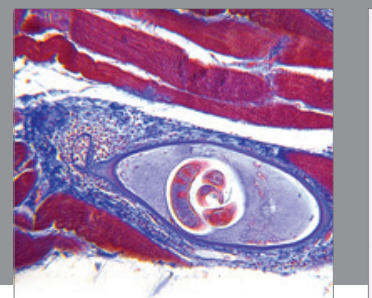

Gastroenterology

Research and Practice
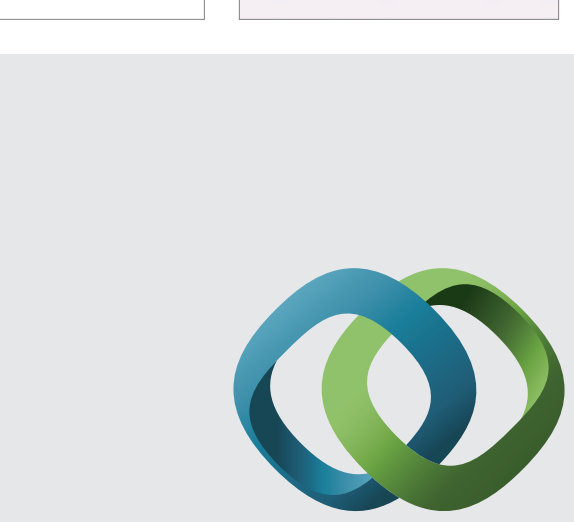

\section{Hindawi}

Submit your manuscripts at

http://www.hindawi.com
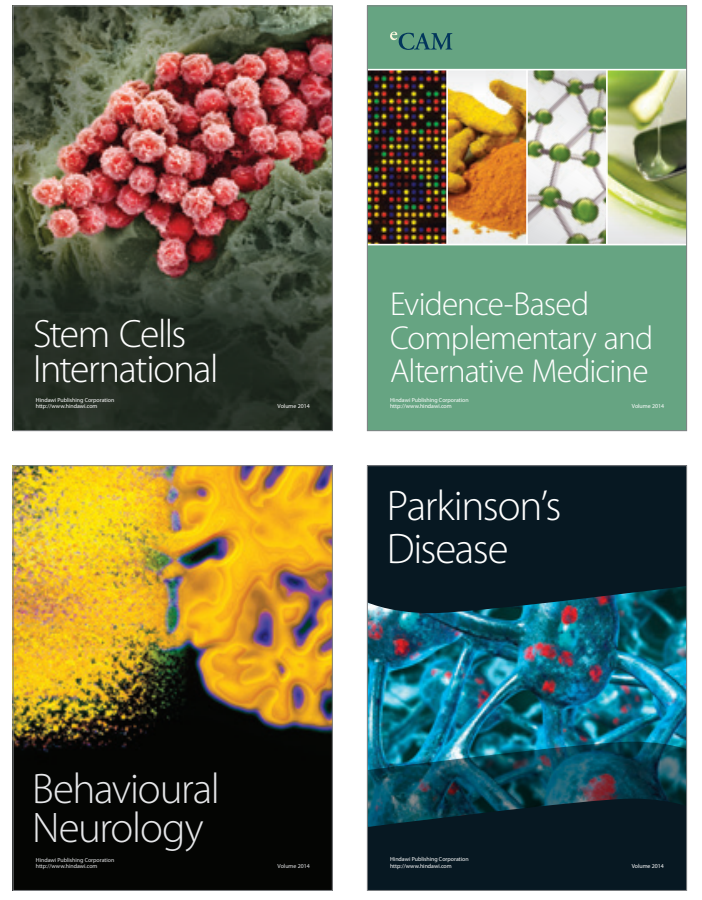
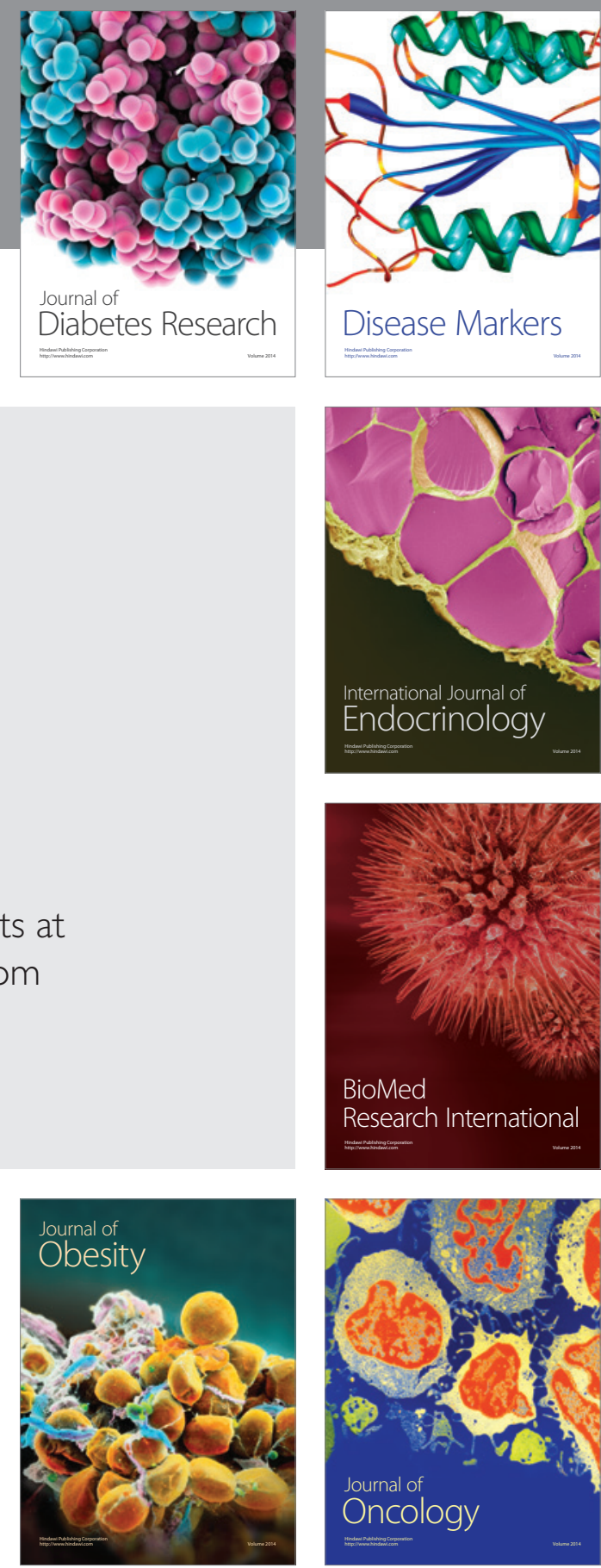

Disease Markers
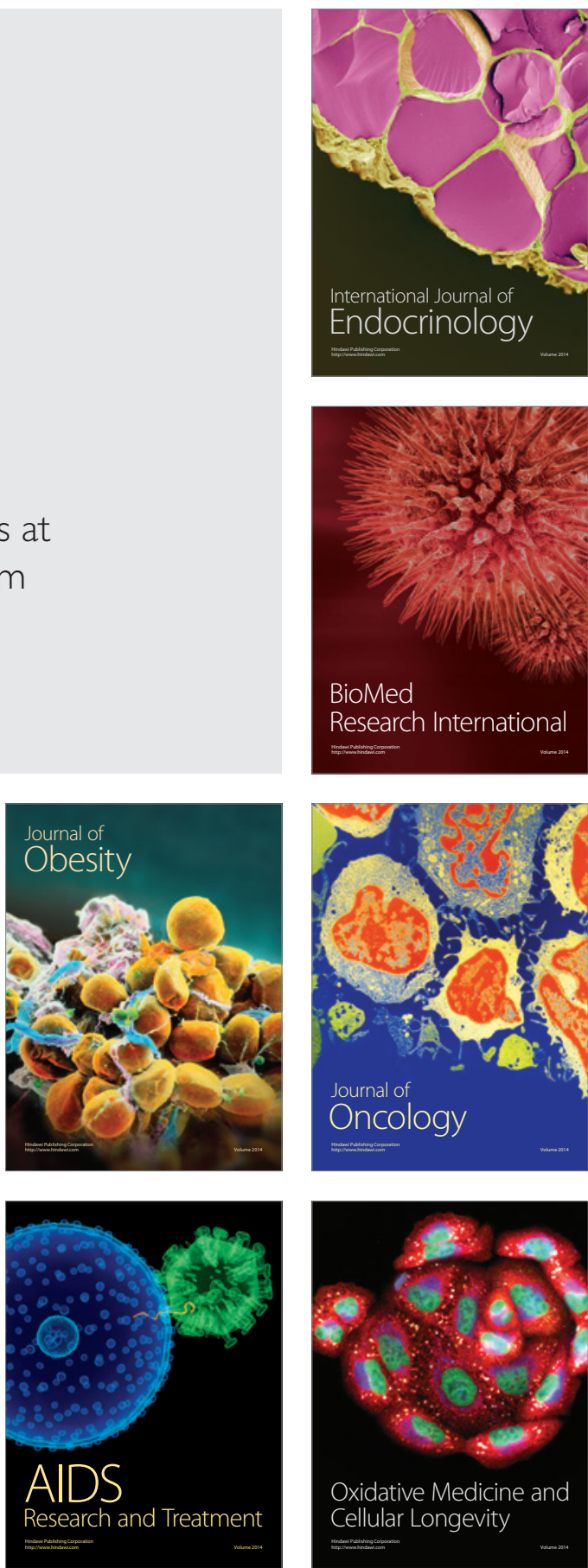